\title{
Baroreflex function in conscious rats submitted to iron overload
}

\section{L.M. Cardoso ${ }^{1}$, \\ M.L. Pedrosa ${ }^{1}$, M.E. Silva ${ }^{2}$, M.F.D. Moraes ${ }^{3}$, E. Colombari ${ }^{4}$ and D.A. Chianca-Jr. ${ }^{1}$}

\author{
Departamentos de ${ }^{1}$ Ciências Biológicas, NUPEB, and ${ }^{2}$ Alimentos, \\ Escola de Nutrição, Universidade Federal de Ouro Preto, Ouro Preto, MG, Brasil \\ ${ }^{3}$ Departamento de Fisiologia e Biofísica, Instituto de Ciências Biológicas, \\ Universidade Federal de Minas Gerais, Belo Horizonte, MG, Brasil \\ ${ }^{4}$ Departamento de Fisiologia, Escola Paulista de Medicina, \\ Universidade Federal de São Paulo, São Paulo, SP, Brasil
}

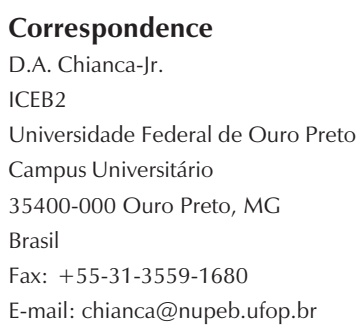

Received December 19, 2003 Accepted October 29, 2004

\begin{abstract}
Our hypothesis is that iron accumulated in tissue, rather than in serum, may compromise cardiovascular control. Male Fischer 344 rats weighing 180 to $220 \mathrm{~g}$ were divided into 2 groups. In the serum iron overload group (SIO, $\mathrm{N}=12$ ), $20 \mathrm{mg}$ elemental iron was injected $i p$ daily for 7 days. In the tissue iron overload group (TIO, $\mathrm{N}=19$ ), a smaller amount of elemental iron was injected ( $10 \mathrm{mg}$, daily) for 5 days followed by a resting period of 7 days. Reflex heart rate responses were elicited by $i v$ injections of either phenylephrine ( 0.5 to $5.0 \mu \mathrm{g} / \mathrm{kg}$ ) or sodium nitroprusside (1.0 to $10.0 \mu \mathrm{g} / \mathrm{kg}$ ). Baroreflex curves were determined and fitted to sigmoidal equations and the baroreflex gain coefficient was evaluated. To evaluate the role of other than a direct effect of iron on tissue, acute treatment with the iron chelator deferoxamine $(20 \mathrm{mg} / \mathrm{kg}$, iv) was performed on the TIO group and the baroreflex was reevaluated. At the end of the experiments, evaluation of iron levels in serum confirmed a pronounced overload for the SIO group (30-fold), in contrast to the TIO group (2-fold). Tissue levels of iron, however, were higher in the TIO group. The SIO protocol did not produce significant alterations in the baroreflex curve response, while the TIO protocol produced a nearly 2 -fold increase in baroreflex gain (-4.34 \pm 0.74 and $-7.93 \pm 1.08 \mathrm{bpm} / \mathrm{mmHg}$, respectively). The TIO protocol animals treated with deferoxamine returned to sham levels of baroreflex gain $(-3.7 \pm 0.3$ sham $v s-3.6 \pm 0.2 \mathrm{bpm} / \mathrm{mmHg}) 30 \mathrm{~min}$ after the injection. Our results indicate an effect of tissue iron overload on the enhancement of baroreflex sensitivity.
\end{abstract}

\section{Introduction}

The cardiovascular system has the crucial task of supplying all body cells with their metabolic needs. The complexity of this function demands efficient control mechanisms for maintaining blood pressure and flux within adequate levels in every region of the body.
Key words

- Baroreflex

- Iron overload

- Heart rate

- Blood pressure

- Iron dextran

- Deferoxamine 
known to alter the responsiveness of the baroreflex response (2).

Iron overload is associated with several pathophysiological states (e.g., hemoglobinopathies, hemolytic anemia, hemochromatosis) and behaviors such as smoking and excessive iron and vitamin C intake (3). Besides the epidemiologic concern regarding dietary iron intake, iron accumulation and its relation to pathological processes is still the main focus of many heated debates (4). Nevertheless, iron overload has undeniably been associated with cardiac and vascular dysfunctions in experimental conditions (5-7). Experiments conducted ex vivo have shown that iron overload induces a condition of heart failure (8) in Langendorff preparations of isolated hearts. In fact, deposition of iron into the heart is emerging as an important cause of heart failure (6). However, a detailed study of cardiovascular reflex involvement in an in vivo iron overload model has not yet been reported. Such an investigation could contribute to the use of iron overload as an animal model for studying heart failure.

Iron has been associated with the modulation of the metabolism and availability of certain chemical mediators such as nitric oxide (9-11) and carbon monoxide (12), which are implicated in baroreflex function both peripherally and centrally (13-15). Nitric oxide as well as oxygen-derived free radicals induced by changes in iron concentration are known to suppress baroreceptor activity particularly at high levels of arterial pressure (15). Recent studies have demonstrated alterations in baroreflex function arising from processes that produce free radicals such as atherosclerosis, in which the baroreflex gain coefficient was shown to be decreased (16). Although cardiac insufficiency is more closely related to the inadequacy of the heart to function as a pump, dysfunction of neural feedback mechanisms (e.g., baroreflex) has been associated with chronic heart failure. Increased sympathetic activity and plasma levels of norepinephrine, parasympathetic withdrawal and impaired baroreflex gain coefficient have been reported in chronic heart failure (17). An increased baroreflex gain coefficient has been shown in rats with myocardial infarction (18). Very few data are available regarding the prognostic implications of baroreflex sensitivity and heart rate (HR) variability for chronic heart failure. Nevertheless, both baroreflex sensitivity and HR variability have been shown to be markedly reduced in chronic heart failure and significantly associated with the degree of ventricular dysfunction and with further progression of the severity of the disease (17). Thus, the impairment of baroreflex function in patients with heart failure is a well-established association. In fact, the evaluation of baroreflex sensitivity has been proposed as a diagnostic tool in such cases (19).

Our hypothesis is that iron overload could affect baroreflex function through iron accumulation in the heart tissue. Therefore, the net effect of iron overload on the baroreflex, as demonstrated in the reports cited above, may not be related to neural control but rather to heart impairment. The present experiment was designed to compare the effect of circulating iron overload to that of iron accumulated in tissues on the baroreflex of conscious Fischer 344 rats. The effect of acute iron chelator treatment (deferoxamine) in the issue iron overload group described above was also tested.

\section{Material and Methods}

\section{Animals and iron overload}

The study was conducted on male Fischer 344 rats weighing 180 to $210 \mathrm{~g}$ from the main breeding stock of the Nutrition School, Federal University of Ouro Preto. Animals were kept in collective cages on a 12-h light/ dark cycle, with lights on at 7:00 am, in a controlled-temperature environment $(22 \pm$ 
$3^{\circ} \mathrm{C}$ ). Animals were randomly divided into the following groups: serum iron overload group (SIO), tissue iron overload group (TIO) (20), and respective controls. In the SIO protocol $(\mathrm{N}=12), 20 \mathrm{mg}$ elemental iron (0.2 ml iron dextran; Sigma, St. Louis, MO, USA) was injected $i p$ daily for 7 days, immediately followed by baroreflex evaluation. In the TIO protocol $(\mathrm{N}=19)$, less elemental iron was injected (10 mg, daily) for a shorter period of time ( 5 days), followed by a resting period of 7 days, after which the experiments were conducted. Sham animals $(\mathrm{N}=$ 31) received placebo injections (PBS) according to their respective iron-loaded groups. Seven animals from the TIO proto$\mathrm{col}$, along with their respective controls ( $\mathrm{N}=$ 7), were separated to receive deferoxamine mesylate (DFO, $20 \mathrm{mg} / \mathrm{kg}$; Novartis AG, Basel, Switzerland), in order to determine the effect of permanent changes in baroreflex function compared to the effect of the direct action of iron. Efforts were made to avoid any unnecessary distress to the animals, in accordance with the Brazilian Society for Neuroscience and Behavior Guidelines for Animal Experimentation.

\section{Surgical procedures and hemodynamic measurements}

Under tribromoethanol anesthesia (250 $\mathrm{mg} / \mathrm{kg}$, ip; Aldrich Chemical Company, Inc., Milwaukee, WI, USA) a heat-pulled taperending polyethylene catheter (PE-10 connected to PE-50; Clay Adams, Parsippany, NJ, USA) filled with heparinized PBS (125 $\mathrm{U} / \mathrm{ml}$ ) was positioned inside the aorta through the left femoral artery for measurement of pulsatile arterial pressure. A second catheter was inserted into the inferior vena cava through the left femoral vein for systemic drug administration. The free endings of both catheters were tunneled subcutaneously and exteriorized through the back of the neck and connected to a swivel during the experiments. Pulsatile arterial pressure was meas- ured with a pressure transducer (model MLT0699; ADInstruments Pty Ltd., Castle Hill, NSW Australia) connected to an analog-to-digital data acquisition system (model PowerLab 400; ADInstruments). Data were sampled at 12 bits using a $200-\mathrm{Hz}$ sampling rate. HR and mean arterial pressure (MAP) were derived off-line from pulsatile arterial pressure using the Chart for Windows software, version 4.1.2 (ADInstruments). HR and MAP variability was estimated by calculating the standard deviation of HR and MAP values within the recording for each animal. All experiments were performed approximately $24 \mathrm{~h}$ after surgery on unanesthetized freely moving rats.

\section{Baroreflex activation}

Before the experiments, rats were instrumented and allowed to adapt to the new environment for at least $30 \mathrm{~min}$. A 20- to 30min recording period without any interference was allowed to elapse in order to determine baseline MAP and HR values.

A typical recording procedure consisted of continuously monitoring HR and MAP while performing intravenous bolus injections of random doses of either phenylephrine $(0.5,2.0,3.5$, and $5.0 \mu \mathrm{g} / \mathrm{kg}$; Sigma) or sodium nitroprusside (1.0, 4.0, 7.0, and 10.0 $\mu \mathrm{g} / \mathrm{kg}$; Sigma). The methodology has been described in detail elsewhere (18). Successive drug administration was carried out only after MAP and HR returned to baseline.

Peak values of MAP and HR in response to phenylephrine and sodium nitroprusside injections were used to evaluate the baroreflex response. The data from each experimental group were then organized in a twocolumn table with data ordered from the lowest to the highest MAP, along with the respective HR values. These data were divided into $10 \mathrm{mmHg}$ pressure bins. For each pressure bin, one MAP \pm SEM value was plotted against its respective HR \pm SEM value. The baroreflex curve was then fitted 
to a sigmoidal logistic equation:

$$
H R=H R_{\max }+\frac{H R_{\min }-H R_{\max }}{1+e^{-\left(\frac{M A P-M A P_{50}}{b}\right)}}
$$

where $H R_{\text {min }}$ is the lower plateau, $H R_{\max }$ is the upper plateau, $b$ is the curvature coefficient, and $M A P_{50}$ is the MAP at the midpoint of the HR range.

Individual sigmoidal functions were averaged in order to determine the mean sigmoidal fit for the group. The derivatives of individual sigmoidal baroreflex curves were calculated and averaged within an experimental group in order to determine the baroreflex gain-coefficient curve.

\section{Acute deferoximine treatment}

Experiments with the chelator DFO were carried out on 7 animals from the TIO protocol and respective controls $(\mathrm{N}=7)$. The baroreflex was evaluated by intravenous bolus injection of sodium nitroprusside (1.0 and $4.0 \mu \mathrm{g} / \mathrm{kg}$; Sigma) before and after $i v$ infusion of DFO $(20 \mathrm{mg} / \mathrm{kg})$. The maximal changes in MAP and HR were evaluated and the gain coefficient was calculated by the equation:

Gain $=\frac{\Delta_{\max } H R}{\Delta_{\max } M A P}$

where $\Delta_{\max } M A P$ is the maximal change in MAP after injection of sodium nitroprusside and $\Delta_{\max } H R$ is the maximal change in HR due to the changes in MAP caused by the pharmacological maneuver.

\section{Determination of iron status}

At the end of the experiments, a blood sample was taken through the arterial cannula and centrifuged and serum separated. Serum iron concentrations were determined in non-hemolyzed serum samples by spectrophotometric analysis using commercially available kits (\# 38; Labtest, Belo Horizonte, MG, Brazil). Immediately after blood sampling, the animals were sacrificed with ethyl ether and perfused with saline and formaldehyde. The liver and spleen were removed and weighed and liver and spleen samples were homogenized in $\mathrm{HNO}_{3}$ at $120^{\circ} \mathrm{C}$. After evaporation of the acid, the dry residue was resuspended in $50 \%$ hydrochloric acid (v/v) and quantified by colorimetric analysis using orthophenanthroline (21).

\section{Statistical analysis}

Data are reported as means \pm SEM. The baroreflex curves were analyzed by one-way analysis of variance (ANOVA) for repeated measures. The unpaired Student $t$-test was used to determine statistically significant differences between the SIO and TIO experimental groups. The paired Student $t$-test was used to determine the statistically significant differences in the TIO baroreflex gain coefficient before and after DFO treatment. The level of significance was set at $\mathrm{P}<0.05$ in all analyses.

\section{Results}

Iron status and baseline cardiovascular parameters

The iron status of the animals treated with iron dextran confirmed our expectations regarding the effect of serum and tissue iron overload on the baroreflex response (Table 1), i.e., the SIO group presented very high serum levels of iron (29-fold) compared to the modest increase observed in the TIO group (1.8-fold). On the other hand, tissue accumulation (e.g., spleen and liver) of iron was much more evident in the TIO group, even though less iron was injected when compared to the SIO animals.

Both iron-treated groups showed a slight, but significant, bradycardia compared to the sham group (SIO protocol: $341 \pm 4 v s 373 \pm 5$ 
bpm; TIO protocol: $354 \pm 7$ vs $373 \pm 5$ bpm). However, baseline MAP values were not significantly different between groups (Figure 1).

A slight decrease in pulse pressure was observed in TIO rats compared to sham rats $(37.9 \pm 0.92$ vs $40.5 \pm 0.85 \mathrm{mmHg}, \mathrm{P}=$ 0.034; data not shown). SIO rats did not present alterations in pulse pressure when compared with sham rats $(37.0 \pm 0.89$ vs $38.8 \pm 0.94 \mathrm{mmHg}, \mathrm{P}=0.186$; data not shown). The HR time domain variability was decreased in both iron-loaded groups compared to the sham group (SIO protocol: $14 \pm 1$ vs $19 \pm 2 \mathrm{bpm}$; TIO protocol: $13 \pm 1$ vs $19 \pm 2 \mathrm{bpm})$. No statistically significant differences were observed in MAP time domain variability (Figure 2 ).

\section{Baroreflex}

The upper panels in Figure 3 show the MAP against HR baroreflex curve, along with the mean sigmoidal fitting, for SIO (Figure 3A) and TIO (Figure 3B) rats and their respective controls. The baroreflex gain coefficient versus pressure plot is reported in the lower panels in Figure 3 for both SIO (Figure 3C) and TIO (Figure 3D) animals.

The baroreflex curve for the TIO group was shifted to the left (Figure 3B,D), closely following the changes in resting MAP (Figure 1 ), as indicated by the $\mathrm{MAP}_{50}$ value. No significant changes were observed in the baroreflex curve of SIO rats compared to control (Figure 3A,C). Maximal reflex tachycardia (upper plateau) and bradycardia (lower plateau), and consequently HR range, for the two iron-treated groups did not differ statistically from control (Table 2). However, a tendency to a reduction in both upper plateau and HR range was noted in SIO rats when compared to sham rats (Table 2, Figure 3A).

TIO rats presented an increased baroreflex gain-coefficient (1.6-fold over control) with a concomitant narrowing of the curve accompanied by a shift to lower MAP values (Table 2, Figure 3B,D).
Table 1. Iron status of sham, serum iron overload and tissue iron overload rats.

\begin{tabular}{lccc}
\hline Parameter & Sham & SIO & TIO \\
\hline Serum iron ( $\mu \mathrm{mol} / \mathrm{l})$ & $35.6 \pm 1.97$ & $1032.3 \pm 209.68^{*}$ & $64.9 \pm 3.94^{*+}$ \\
Liver iron $(\mu \mathrm{mol} /$ organ) & $46.6 \pm 9.49$ & $91.3 \pm 15.8^{*}$ & $164.7 \pm 10.74^{*+}$ \\
Spleen iron ( $\mu \mathrm{mol} /$ organ) & $20.6 \pm 1.20$ & $19.7 \pm 2.00$ & $30.1 \pm 1.20^{*+}$
\end{tabular}

Data are reported as means \pm SEM for 8 rats in each group. SIO = serum iron overload; $\mathrm{TIO}=$ tissue iron overload.

${ }^{*} \mathrm{P}<0.05$ compared to the sham group; ${ }^{+P}<0.05$ compared to the SIO group (Student $t$-test).

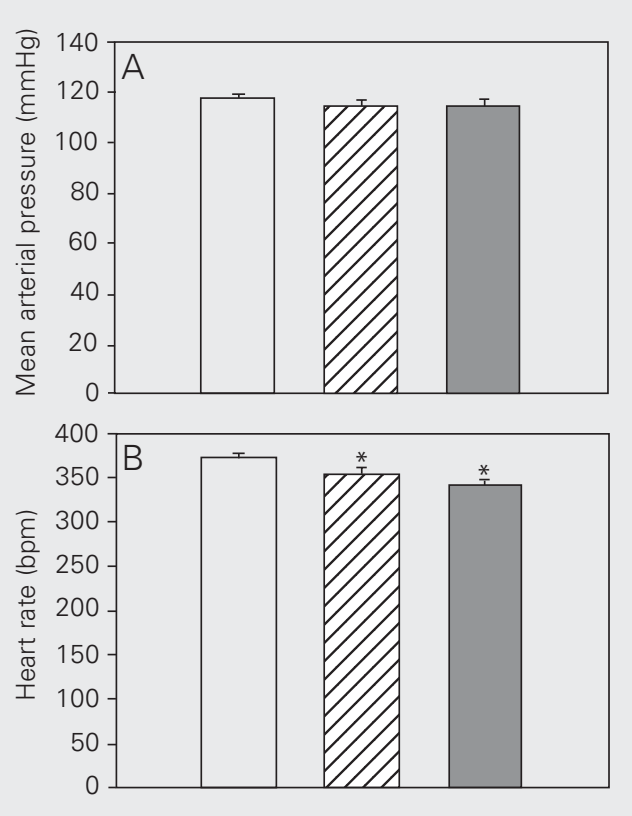

Figure 1. Baseline mean arterial pressure $(A)$ and heart rate $(B)$ of sham (open bar, $\mathrm{N}=24$ ), tissue iron overload (hatched bar, $\mathrm{N}=19$ ) and serum iron overload (filled bar, $N=12$ ) rats over a period of $10 \mathrm{~min}$ at the beginning of the recordings. Data are reported as mean \pm SEM. ${ }^{*} \mathrm{P}<$ 0.05 compared to the sham group (Student $t$-test).

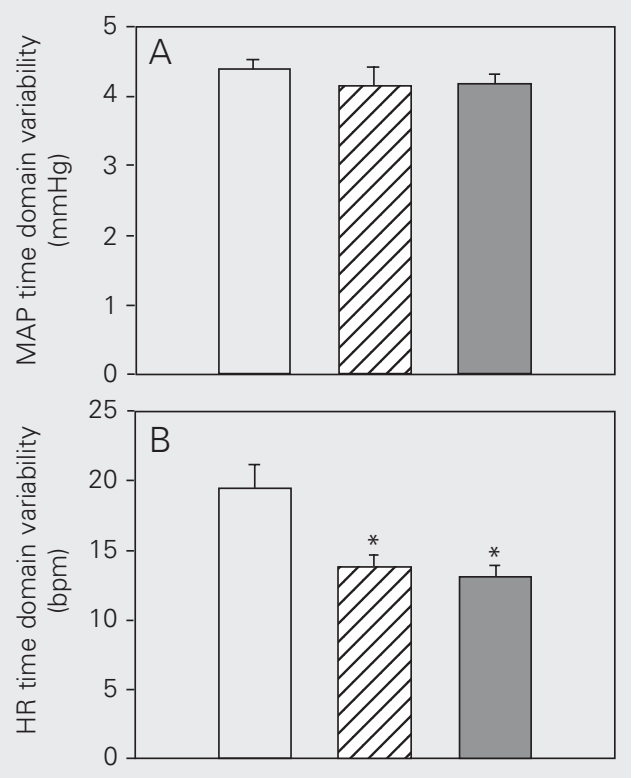

Figure 2. Time domain variability for mean arterial pressure (A) and heart rate $(B)$ in sham (open bar, $N=24$ ), tissue iron overload (hatched bar, $N=19$ ) and serum iron overload (filled bar, $N=12$ ) rats over a period of 10 min at the beginning of the recordings. Data are reported as means \pm SEM. MAP $=$ mean arterial pressure; $H R=$ heart rate. ${ }^{*} P<0.05$ compared to the sham group (Student $t$ test). 


\section{Effects of acute deferoximine treatment}

The upper panel in Figure 4 represents the gain coefficient of the baroreflex tachy-

Figure 3. The upper panels (A and $B$ ) show peak values of mean arterial pressure and heart rate after baroreflex stimulation with phenylephrine and sodium nitruprusside. The broken lines and open circles represent irontreated rats and the continuous line and filled circles represent sham rats. The data for serum iron overload $(\mathrm{SIO})$ rats are illustrated in the panels on the left (A and $\mathrm{C}$ ) and the data for tissue iron overload (TIO) rats are illustrated in the panels on the right ( $B$ and $D)$. The logistic sigmoidal-fitting baroreceptor curve is the mean of ten sigmoids fitted from the data for conscious SIO rats $(N=12)$, TIO rats $(N=12)$ and respective sham $(N=12)$ rats. The lower panels indicate baroreflex gain, e.g., the first derivative of the sigmoid functions, at specific mean arterial pressure values.

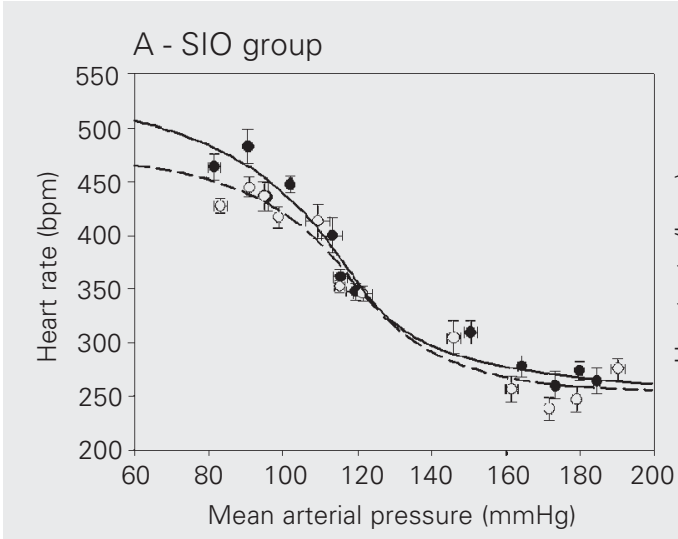

cardia (Equation 2) for the TIO and sham rats. The MAP range in which the baroreflex gain coefficient was evaluated corresponds to the linear portion of the baroreflex curve
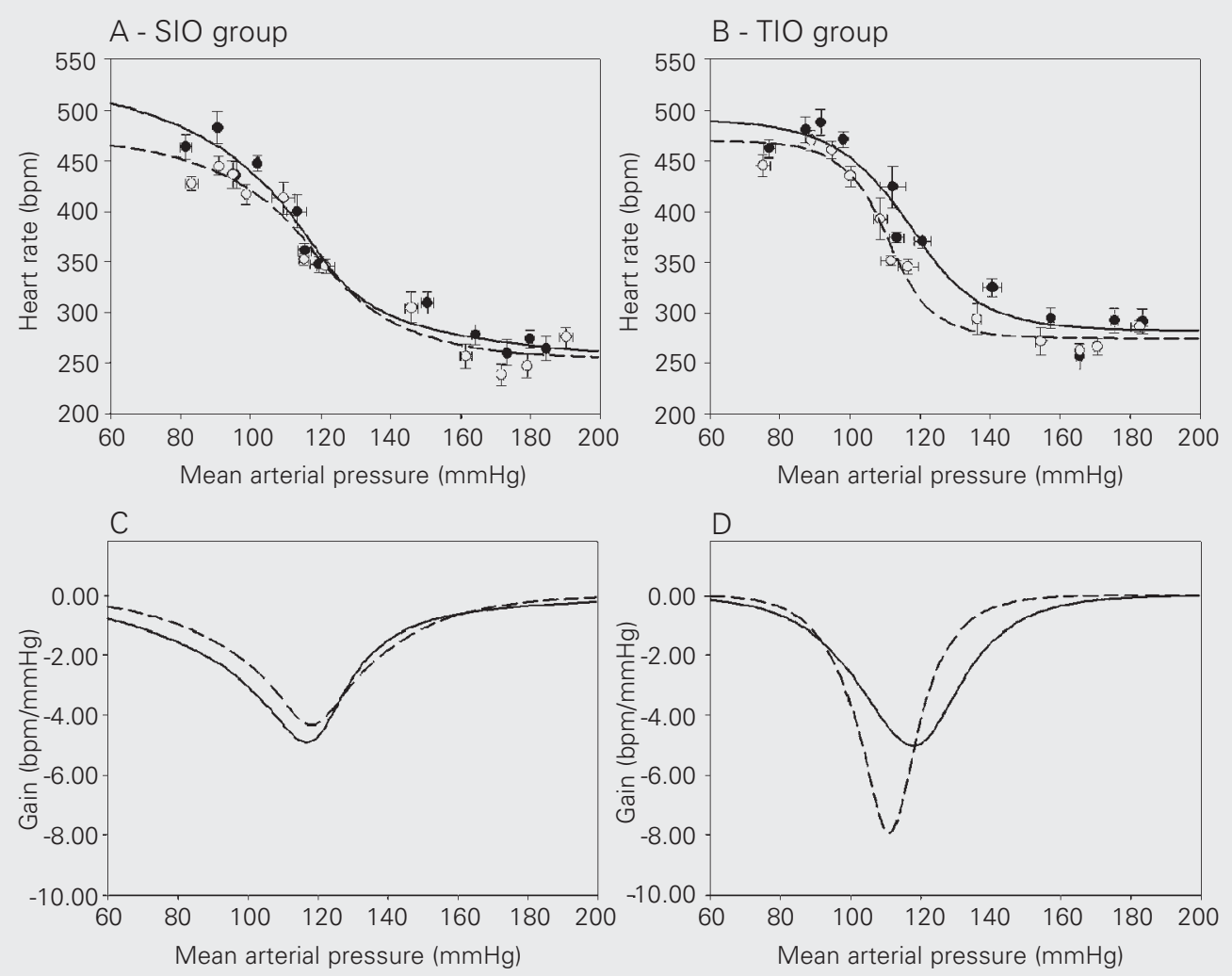

- Sham

- SIO group

_ Mean fitting from sham group

Mean fitting from $\mathrm{SIO}$ group
- Sham

- TIO group

_ Mean fitting from sham group Mean fitting from $\mathrm{TIO}$ group

Table 2. Baroreflex curve parameters of sham, serum iron overload and tissue iron overload rats.

\begin{tabular}{lcccc}
\hline \multirow{2}{*}{ Parameter } & \multicolumn{2}{c}{ SIO } & & TIO \\
\cline { 2 - 5 } & Sham & Iron treated & Sham & Iron treated \\
\cline { 2 - 5 } & & & \\
Upper plateau (bpm) & $528 \pm 27$ & $473 \pm 11$ & $492 \pm 11$ & $469 \pm 9$ \\
Lower plateau (bpm) & $253 \pm 10$ & $254 \pm 5$ & $282 \pm 5$ & $274 \pm 5$ \\
Heart rate range (bpm) & $275 \pm 27$ & $218 \pm 9$ & $210 \pm 13$ & $196 \pm 9$ \\
MAP $(\mathrm{mmHg})$ & $114 \pm 4$ & $117 \pm 3$ & $117 \pm 1$ & $111 \pm 1^{*}$ \\
Gain (bpm/mmHg) & $-4.91 \pm 0.69$ & $-4.34 \pm 0.74$ & $-5.03 \pm 0.39$ & $-7.93 \pm 1.08^{*}$
\end{tabular}

Data are reported as means \pm SEM for 12 rats in each group. SIO = serum iron overload; $\mathrm{TIO}=$ tissue iron overload; $\mathrm{MAP}_{50}=$ mean arterial pressure at the midpoint of the heart rate range.

${ }^{*} P<0.05$ compared to the sham group (Student $t$-test). 
(Figure 4, lower panel; copied from Figure $3 \mathrm{~B}$ indicating the MAP range).

Infusion of the free iron chelator (DFO) reduced the baroreflex gain values of the TIO rats (Table 3$)$ to control levels (5.28 \pm 0.86 vs $3.57 \pm 0.27 \mathrm{bpm} / \mathrm{mmHg}$ ) but did not affect the baroreflex gain coefficient in sham animals $(3.77 \pm 0.40$ vs $3.66 \pm 0.41 \mathrm{bpm} /$ $\mathrm{mmHg}$ ) when baroreflex was evaluated by sodium nitroprusside injection.

\section{Discussion}

In this study, we sought to determine whether iron overload produced by intraperitoneal injections of iron dextran affects baroreflex function in conscious rats. The iron profile of SIO and TIO rats confirmed our expectation (Table 1) of an experimental model that would compare the effect of high serum iron levels with the long-term effects of iron accumulated in tissue. The results demonstrated that the baroreflex gain was increased in TIO rats (Figure 3), whereas no differences in baroreflex function were observed in SIO rats. Furthermore, the data indicate a direct effect of iron in tissue, since DFO, a chelator, was able to reverse the phenomenon (Figure 4). Therefore, the present data support the view that iron overload plays a role in the functional changes regarding the effector components of baroreflex function, as suggested previously by others (22).

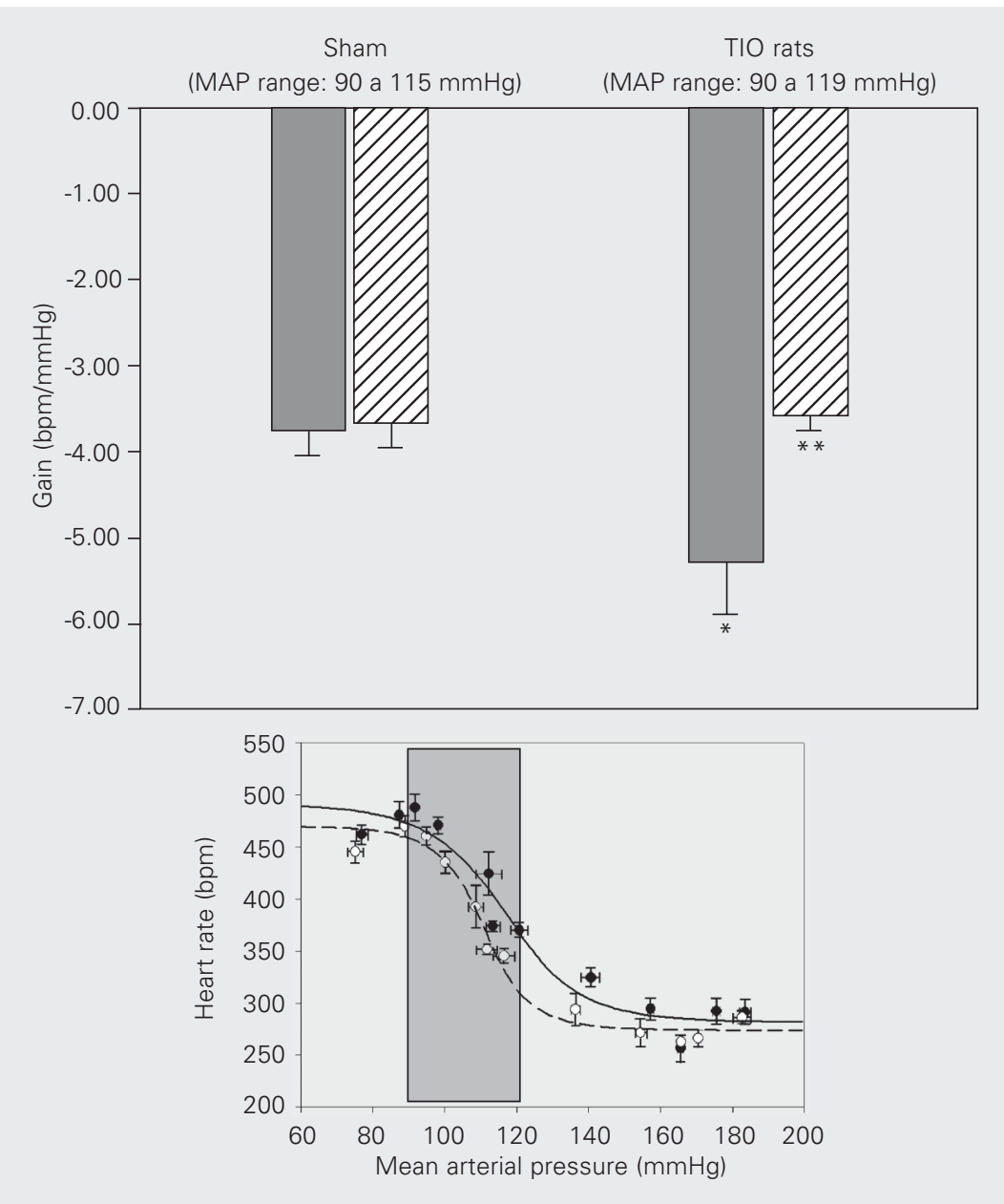

Figure 4. General baroreflex gain elicited before (filled bar) and after (hatched bar) deferoxamine infusion $(20 \mathrm{mg} / \mathrm{kg}$, over $20 \mathrm{~min})$. Left bars indicate sham rats and right bars indicate tissue iron overload (TIO) rats. The bottom panel is a copy of Figure 3B with the marked area showing the linear region of the baroreflex curve in which baroreflex was tested in the deferoxamine experiment. ${ }^{*} P<0.05$ compared to the sham group; ${ }^{*} P<0.05$ compared to the TIO rats before deferoxamine infusion (Student $t$-test).

Table 3. Changes in heart rate, mean arterial pressure and mean gain values of baroreflex evaluated by sodium nitroprusside before and after deferoxamine $(20 \mathrm{mg} / \mathrm{kg})$ infusion in sham and tissue iron overload rats.

\begin{tabular}{|c|c|c|c|c|c|c|c|c|c|c|c|c|}
\hline \multirow[t]{3}{*}{ Group } & \multicolumn{6}{|c|}{ Before DFO } & \multicolumn{6}{|c|}{ After DFO } \\
\hline & \multicolumn{3}{|c|}{$1.0 \mu \mathrm{g} / \mathrm{kg} \mathrm{NP}$} & \multicolumn{3}{|c|}{$4.0 \mu \mathrm{g} / \mathrm{kg} \mathrm{NP}$} & \multicolumn{3}{|c|}{$1.0 \mu \mathrm{g} / \mathrm{kg} \mathrm{NP}$} & \multicolumn{3}{|c|}{$4.0 \mu \mathrm{g} / \mathrm{kg} \mathrm{NP}$} \\
\hline & $\Delta \mathrm{HR}$ & $\triangle \mathrm{MAP}$ & Gain & $\Delta \mathrm{HR}$ & $\triangle \mathrm{MAP}$ & Gain & $\Delta \mathrm{HR}$ & $\triangle \mathrm{MAP}$ & Gain & $\Delta \mathrm{HR}$ & $\triangle \mathrm{MAP}$ & Gain \\
\hline Sham & $79 \pm 6$ & $-20 \pm 2$ & $4.24 \pm 0.43$ & $94 \pm 5$ & $-29 \pm 2$ & $3.30 \pm 0.29$ & $81 \pm 5$ & $-21 \pm 2$ & $4.02 \pm 0.45$ & $89 \pm 7$ & $-28 \pm 2$ & $3.30 \pm 0.34$ \\
\hline TIO & $86 \pm 11$ & $-14 \pm 1^{*}$ & $6.53 \pm 1.01$ & $89 \pm 9$ & $-22 \pm 2$ & $4.08 \pm 0.24$ & $85 \pm 8$ & $-26 \pm 3$ & $3.44 \pm 0.32$ & $99 \pm 11$ & $-27 \pm 2$ & $3.71 \pm 0.26$ \\
\hline
\end{tabular}

Data are reported as means $\pm \mathrm{SEM}$ for 7 rats in each group. $\mathrm{HR}=$ heart rate; $\mathrm{MAP}=$ mean arterial pressure; $\mathrm{DFO}=$ deferoxamine mesylate; $\mathrm{NP}=$ sodium nitroprusside; $\mathrm{TIO}=$ tissue iron overload.

${ }^{*} \mathrm{P}<0.01$ compared to the sham group (Student $t$-test). 
Chronic iron overload produces heart dysfunction in a dose-dependent manner (8), probably resulting from the time lag necessary to deposit iron in tissues. In the heart, iron, initially deposited in the epicardium, has a long term-effect on transmural wall thickness $(23,24)$. Such long-term effects do not seem to have occurred in TIO rats since DFO was able to acutely reverse the effect of iron overload on the baroreflex gain coefficient. The interpretation of the short-term and long-term effects of iron in the present study is compromised by the lack of published information concerning acceptable levels of iron in the rat. Neither the borderline levels of iron nor the exposure time needed in order to produce iron-related dysfunctions in the cardiovascular system have been reported. Nevertheless, several reports have demonstrated the possible role of cardiac iron deposition in the development of cardiomyopathy and heart failure within the iron overload paradigm $(23,25)$.

On the basis of the Haber-Weiss and Fenton reactions, iron can produce hydroxyl radicals that might lead to oxidative stress. Extensive reviews about the effect of iron, even at low concentrations, on free radicals and calcium homeostasis are available (2629). A dose-dependent effect of oxidative damage in rats submitted to the iron overload paradigm (30) agrees with our findings of the stronger cardiovascular effects encountered in TIO rats. Since deferoxamine chelates iron ions and limits the Haber-Weiss and Fenton reactions, preventing the formation of hydroxyl radicals, it becomes impossible to dissociate a direct effect of iron on cardiac tissue from that of free radicals. Thus, further studies using specific free radical scavengers should be carried out to elucidate the mechanism by which DFO was able to reverse the effect of the TIO protocol on the baroreflex gain coefficient.

Factors that modulate calcium homeostasis in cardiomyocytes affect both contractility and cardiac rhythm. The proper control of calcium inward current in myocytes is essential for normal electrical rhythms, while its dysfunction may generate life-threatening heart arrhythmias (31) along with other pathological conditions. Both ferrous ions $\left(\mathrm{Fe}^{2+}\right)$ and free radicals have been associated with the impairment and breakdown of $\mathrm{Ca}^{2+}$ ATPase proteins in the sarcoplasmic reticulum of the rat heart (26). Oxidative stress has been suggested to enhance calcium currents through neuronal $\mathrm{Ca}^{2+}$ channels (27). The heart has a high density of L-type $\mathrm{Ca}^{2+}$ channels, which are directly modulated by $\mathrm{Fe}^{2+}$ ions (28). In addition, myocardial $\mathrm{Fe}^{2+}$ uptake has been reported to occur via L-type $\mathrm{Ca}^{2+}$ channels (29), indicating a complex interaction between calcium, iron and free radical generation in the heart and neuronal tissue. In synthesis, both excess of iron and free radicals have been implicated in the disruption of intracellular calcium homeostasis $(32,33)$ not only in cardiomyocytes but also in neurons $(34,35)$. Taken together, these reports agree with the hypothesis that the increase in baroreflex tachycardia (Figure 3) could represent an adjustment process to compensate for iron-induced failure in myocardial function, sustaining, at least in part, blood pressure within normal levels. In fact, the narrowing of the baroreflex curve, the increase in baroreflex gain coefficient and the left shift of the $\mathrm{MAP}_{50}$ (Figure 3B and D) suggest myocardial rather than neural dysfunction. In addition, our data from the baroreflex curve profiles of TIO rats very much resemble data reported by Meyrelles and collaborators (18) using an animal model of heart failure induced by occlusion of the anterior descending coronary artery. In their study, these investigators found that although the baroreflex gain coefficient was unaltered after the first day of infarction, 30 days after occlusion the baroreflex gain was significantly exaggerated. Still regarding the abovementioned report, one cannot fail to notice the striking similarities between the baroreflex curves and gain curves presented here 
(for the SIO and TIO protocols; Figure 3) and those of 1- and 30-day infarcted animals (18). Nevertheless, the effects of iron overload on other factors that modulate calcium homeostasis in cardiomyocytes cannot be ruled out, such as $\mathrm{Ca}^{2+}$-ATPase pump involvement, low levels of $\mathrm{Ca}^{2+}$ in the reticular sarcoplasm, $\mathrm{Ca}^{2+}$ voltage-dependent channel sensitivity, or ryanodine receptor expression, among others $(36,37)$.

Our results support the idea of a peripheral action of iron affecting baroreflex function, e.g., an iron-dependent impairment of myocardial function associated with heart failure, since DFO was able to reverse the effects of altered baroreflex gain observed in Figure 3. Bartfay and collaborators (6) reported finding a decrease of baseline HR using the Langendorff isolated heart perfusion technique in iron dextran-treated mice. Our in vivo findings and Bartfay's ex vivo findings for baseline HR could be due to the same intrinsic mechanisms and could result, at least in part, from impairment of calcium channels due to the action of free radicals or to a direct action of ferrous iron. The decrease in pulse pressure observed in TIO rats can be interpreted as a possible consequence of the reduction of heart strength by ironinduced heart failure.

Our results support the hypothesis of ironinduced heart failure as an important factor for the development of the observed adjustment in baroreflex function. The data also indicate that the iron overload model is an interesting in vivo model for heart failure that is both gradual and, to some extent, reversible. Although we do not exclude the possibility of an involvement of free radicals and/or iron in the adjustments of the afferent baroreflex pathway, the data strongly indicate that the baroreflex curve change is the neural response to myocardial incapacity to properly respond to central control.

\section{References}

1. Machado BH, Mauad H, Chianca-Jr DA, Haibara AS \& Colombari $E$ (1997). Autonomic processing of the cardiovascular reflexes in the nucleus tractus solitarii. Brazilian Journal of Medical and Biological Research, 30: 533-543.

2. Head GA (1994). Cardiac baroreflexes and hypertension. Clinical and Experimental Pharmacology and Physiology, 21: 791-802.

3. Weinberg ED (1990). Cellular iron metabolism in health and disease. Drug Metabolism Reviews, 22: 531-579.

4. Beard J (2002). Dietary iron intakes and elevated iron stores in the elderly: is it time to abandon the set-point hypothesis of regulation of iron absorption? American Journal of Clinical Nutrition, 76: 11891190.

5. Asimakis GK, Inners KF \& Ethridge RT (1997). Role of low molecular weight iron in functional preconditioning of the isolated rat heart. Journal of Molecular and Cellular Cardiology, 29: 1087-1096.

6. Bartfay WJ, Dawood F, Wen WH, Lehotay DC, Hou D, Bartfay E, Luo X, Backx PH \& Liu PP (1999). Cardiac function and cytotoxic aldehyde production in a murine model of chronic iron overload. Cardiovascular Research, 43: 892-900.

7. Crawford RD (1995). Proposed role for a combination of citric acid and ascorbic acid in the production of dietary iron overload: a fundamental cause of disease. Biochemistry and Molecular Medicine, 54: 1-11

8. Bartfay WJ \& Bartfay E (2000). Iron overload cardiomyopathy: evidence for a free radical-mediated mechanism of injury and dysfunction in a murine model. Biological Research for Nursing, 2: 49-59.

9. Chen L, Wang $Y$, Kairaitis LK, Wang $Y$, Zhang BH \& Harris DC
(2001). Molecular mechanisms by which iron induces nitric oxide synthesis in cultured proximal tubule cells. Experimental Nephrology, 9: 198-204.

10. Takenaka K, Suzuki S, Sakai N, Kassell NF \& Yamada H (1995). Transferrin induces nitric oxide synthase mRNA in rat cultured aortic smooth muscle cells. Biochemical and Biophysical Research Communications, 213: 608-615.

11. Zhou XJ, Laszik Z, Wang XQ, Silva FG \& Vaziri ND (2000). Association of renal injury with increased oxygen free radical activity and altered nitric oxide metabolism in chronic experimental hemosiderosis. Laboratory Investigation, 80: 1905-1914.

12. Anning PB, Chen $Y$, Lamb NJ, Mumby S, Quinlan GJ, Evans TW \& Gutteridge JM (1999). Iron overload upregulates haem oxygenase 1 in the lung more rapidly than in other tissues. FEBS Letters, 447: 111-114.

13. Johnson RA, Kozma F \& Colombari E (1999). Carbon monoxide: from toxin to endogenous modulator of cardiovascular functions. Brazilian Journal of Medical and Biological Research, 32: 1-14.

14. Silva CC, Almeida VA, Haibara AS, Johnson RA \& Colombari E (1999). Role of carbon monoxide in L-glutamate-induced cardiovascular responses in nucleus tractus solitarius of conscious rats. Brain Research, 824: 147-152.

15. Chapleau MW \& Abboud FM (1994). Modulation of baroreceptor activity by ionic and paracrine mechanisms: an overview. Brazilian Journal of Medical and Biological Research, 27: 1001-1015.

16. Li Z, Mao HZ, Abboud FM \& Chapleau MW (1996). Oxygen-derived free radicals contribute to baroreceptor dysfunction in atheroscle- 
rotic rabbits. Circulation Research, 79: 802-811.

17. Mortara A \& Tavazzi L (1996). Prognostic implications of autonomic nervous system analysis in chronic heart failure: Role of heart rate variability and baroreflex sensitivity. Archives of Gerontology and Geriatrics, 23: 265-275

18. Meyrelles SS, Mill JG, Cabral AM \& Vasquez EC (1996). Cardiac baroreflex properties in myocardial infarcted rats. Journal of the Autonomic Nervous System, 60: 163-168.

19. Sack S, Auricchio A, Baumann L, Kadhiresan V, Maarse A, Pochet T \& Kramer A (2000). Baseline baroreflex sensitivity can identify heart failure patients who can benefit from ventricular resynchronization therapy. European Journal of Heart Failure, 2: 13.

20. Turbino-Ribeiro SM, Silva ME, Chianca Jr DA, De Paula H, Cardoso LM, Colombari E \& Pedrosa ML (2003). Iron overload in hypercholesterolemic rats affects iron homeostasis and serum lipids but not blood pressure. Journal of Nutrition, 133: 15-20.

21. Association of Official Analytical Chemists (1980). Official Methods of Analysis. 0-492. AOAC, Washington, DC, USA.

22. Wang W, Han HY \& Zucker IH (1996). Depressed baroreflex in heart failure is not due to structural change in carotid sinus nerve fibers. Journal of the Autonomic Nervous System, 57: 101-108.

23. Liu P \& Olivieri N (1994). Iron overload cardiomyopathies: new insights into an old disease. Cardiovascular Drugs and Therapy, 8: 101-110.

24. Liu P, Henkelman M, Joshi J et al. (1998). Quantification of cardiac and tissue iron by nuclear magnetic resonance relaxometry in a novel murine thalassemia-cardiac iron overload. Canadian Journal of Cardiology, 12: 155-164.

25. Aldouri MA, Wonke B, Hoffbrand AV, Flynn DM, Ward SE, Agnew JE \& Hilson AJ (1990). High incidence of cardiomyopathy in betathalassaemia patients receiving transfusion and iron chelation: reversal by intensified chelation. Acta Haematologica, 80: 113-117.

26. Moreau VH, Castilho RF, Ferreira ST \& Carvalho-Alves PC (1998). Oxidative damage to sarcoplasmic reticulum $\mathrm{Ca}^{2+}$-ATPase at submicromolar iron concentrations: evidence for metal-catalyzed oxidation. Free Radicals in Biology and Medicine, 25: 554-560.

27. Li A, Segui J, Heinemann SH \& Hoshi T (1998). Oxidation regulates cloned neuronal voltage-dependent $\mathrm{Ca}^{2+}$ channels expressed in
Xenopus oocytes. Journal of Neuroscience, 18: 6740-6747.

28. Winegar BD, Kelly R \& Lansman JB (1991). Block of current through single calcium channels by $\mathrm{Fe}, \mathrm{Co}$, and $\mathrm{Ni}$. Location of the transition metal binding site in the pore. Journal of General Physiology, 97: 351-367.

29. Tsushima RG, Wickenden AD, Bouchard RA, Oudit GY, Liu PP \& Backx PH (1999). Modulation of iron uptake in heart by L-type $\mathrm{Ca}^{2+}$ channel modifiers: possible implications in iron overload. Circulation Research, 84: 1302-1309.

30. Lucesoli F, Caligiuri M, Roberti MF, Perazzo JC \& Fraga CG (1999). Dose-dependent increase of oxidative damage in the testes of rats subjected to acute iron overload. Archives of Biochemistry and Biophysics, 372: 37-43.

31. Bers DM (2002). Calcium and cardiac rhythms: physiological and pathophysiological. Circulation Research, 90: 14-17.

32. Burlando B, Panfoli I, Viarengo A \& Marchi B (2001). Free radicaldependent $\mathrm{Ca}^{2+}$ signaling: role of $\mathrm{Ca}^{2+}$-induced $\mathrm{Ca}^{2+}$ release. Antioxidants and Redox Signalling, 3: 525-530.

33. Kim E, Giri SN \& Pessah IN (1995). Iron(II) is a modulator of ryanodine-sensitive calcium channels of cardiac muscle sarcoplasmic reticulum. Toxicology and Applied Pharmacology, 130: 57-66.

34. Lu C, Chan SL, Fu W \& Mattson MP (2002). The lipid peroxidation product 4-hydroxynonenal facilitates opening of voltage-dependent $\mathrm{Ca}^{2+}$ channels in neurons by increasing protein tyrosine phosphorylation. Journal of Biological Chemistry, 277: 24368-24375.

35. Shirotani K, Katsura M, Higo A, Takesue M, Mohri Y, Shuto K, Tarumi C \& Ohkuma S (2001). Suppression of $\mathrm{Ca}^{2+}$ influx through Ltype voltage-dependent calcium channels by hydroxyl radical in mouse cerebral cortical neurons. Molecular Brain Research, 92: 1218.

36 Yasumura Y, Takemura K, Sakamoto A, Kitakazem M \& Miyatakek K (2003). Changes in myocardial gene expression associated with bblocker therapy in patients with chronic heart failure. Journal of Cardiac Failure, 9: 469-473.

37. Yamaguchi F, Sanbe A \& Satoshi T (1997). Cardiac sarcoplasmic reticular function in rats with chronic heart failure following myocardial infarction. Journal of Molecular and Cellular Cardiology, 29: 753763. 